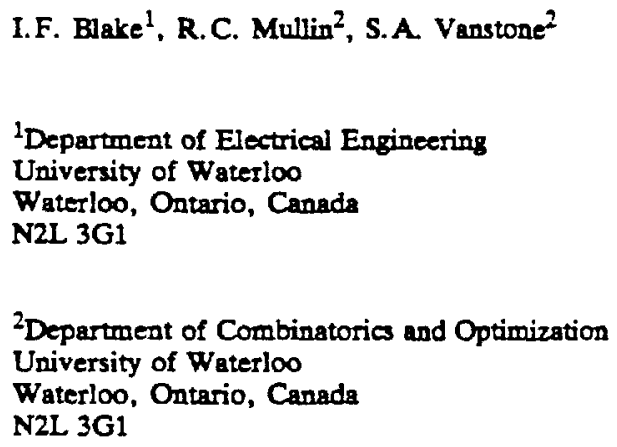

1. The Problem.

Consider the finite field having $q$ elements and denote it by $G F(q)$. Let a be a generator for the nonzero elements of $G F(a)$. Hence, for any element $b \neq 0$ there exist an integer $x, 0 \leq x \leq q-2$, such that $b=\alpha^{x}$. We call $x$ the discrete logarithm of $b$ to the base $\alpha$ and we denote it by $x=\log _{\alpha} b$ and more vimply by $\log b$ when the base is fired for the discussion. The discrete logarithm problem is stated as follows:

Find a computationally feasible algorithm to compute $\log _{a} b$ for any $b \in G F(a), b \neq 0$.

Several cryptographic schemes have been proposed which base their security on the intractability of the discrete logarithm problem for large $q$.

In 1976 Diffie and Hellman [7] proposed the following public key passing scheme. Let $A$ and $B$ be two parties who wish to share a common key $R$. The finite field $G F(q)$ and a generator $a$ are stored in a public file. Party $A$ generates a random integer $a$ and computes $a^{a}$. Party $B$ generates a random integer $b$ and computes $\alpha^{b}$. A sends to $B$ the field element $\alpha^{a}$ and $B$ sends to $A$ the field element $\alpha^{b}$. A computes $\left(a^{b}\right)^{a}=\alpha^{a b}$ and $B$ computes $\left(\alpha^{a}\right)^{b}=\alpha^{a b}$. $A$ and $B$ now share the common key $K=\alpha^{a b}$.

Recently, ElGamel [8] has proposed a public key cyptosystem and an authentication scheme which is based on discrete exponentiation. We describe only the public key system here.

Consider $G F(q)$ generated by $\alpha$. The message space $M$ will consist of all nonzero field elements. Party $A$ generates a random integer $a$ and stores $a^{a}$ in a public file. Suppose party $B$ wishes to send a message $m$ to $A$. $B$ generates a random integer $k$ and computes $\left(a^{a}\right)^{k}$ (since $a^{a}$ is made public this is possible), $a^{k}$ and $m \alpha^{a k}$. B sends $A$ the pair $\left(\alpha^{k}, \alpha^{a k} m\right)$. Since $A$ knows $a$ he can compute $\left(\alpha^{k}\right)^{a}=\alpha^{a k}$, and, hence, obtain $m$ from $a^{a t} m$. This scheme and the Diffie-Hellman method appear to have the same degree of security. It remains an open problem as to whether or not the security of these systems is entirely dependent on comput-

\footnotetext{
Research supported in part by the Departoent of Comminications under contract 205T 36001-4-0853.
} 
ing discrete logarithms.

A number of special purpose algorithms for computing discrete loga have appeared in the literature (see, for example, [2], [9], [11], [14]). In this article we address only the most general methods carrently available.

In the nert three sections we describe three subexponential algorithms for computing logs. The algorithros of sections 3 and 4 are variants of the one presented in section 2. These algorithms are referred to as the index-calculus algorithms in an excellent and in-depth article on the subject by Odlyzko [13].

For the purposes of this paper we restrict our discassion to $G F\left(2^{n}\right)$. (The algorithms of sections 2 and 3 apply more generally.) We think of the elements in $G F\left(2^{n}\right)$ as polynomials of degree at most $n-1$ over $G F(2)$ and multiplication is performed modulo some fired irreducible polynomial of degree $n$ over $G F(2)$. The eramples cited in this paper refer to $G F\left(2^{127}\right.$ ) as it has been of wome interest recently. (See, for example, [15], [17]).

\section{Adleman Algorithm}

The basic ideas involved in the following suberponential algorithm for computing discrete logarithms are due to Western and Miller [16]. Adleman [1] independently discovered the algorithm and partially analysed its computational complexity.

The algorithm consists of two parts. The first part requires the construction of a large database of logarithms. This database only needs to be constructed once for $G F\left(2^{n}\right)$. Part 2 of the algorithm computes individual logarithms.

\section{Part 1 (Database).}

Find the logarithms of all irreducible polynomials of degree at most $b$ where $b$ is a fixed positive integer determined by $G F\left(2^{n}\right)$.

\section{Part 2.}

To find the $\log$ of an element $g(x) \in G F\left(2^{n}\right), g(x) \neq 0$, generate a random integer $a$ and compute $h(x)=g(x) \alpha^{a}(x)$ where $\alpha(x)$ generates $G F\left(2^{n}\right)$. Now, factor

$$
h(x)=\prod_{i=1}^{1} p_{i}^{i_{1}}(x)
$$

If each irreducible factor $p_{i}(x)$ has $\operatorname{deg} p_{i}(x) \leq b$ then

$$
\log g(x)=\sum_{i=1}^{1} e_{i} \log p_{i}(x)-a
$$

which can easily be evaluated by looking up $\log p_{i}(x), 1 \leq i \leq t$, in the database. If not all $p_{i}(x)$ have $\operatorname{deg} p_{i}(x) \leq b$ then generate another random integer and repeat. 
We define $p(n, b)$ to be the probability that a randomly chosen polynomial of degree exactly $n$ has all of its irreducible factors with degrees of at most $b$. If $N(n, b)$ is the number of polynomials of degrees exactly $n$ such that each has all of its irrectucible factorn with degree at most $b$ then

$$
p(n, b)=\frac{N(n, b)}{2^{n}}
$$

and the expected runtime of the second part of the algorithm ahould be approximately $p(n, b)^{-1}$. Odlyzto [1] shows that

$$
p(n, b)^{-1} \equiv\left(\frac{n}{b}\right)^{(1+\alpha(1)) \div}
$$

and that the asymptotic running time of the entire algosithm is $\exp \left(c_{1}(n \log n)^{3 / y}\right)$.

Let $S$ be the set of all irreducible polynomials of degree at most $b$. In order to find the logarithms of all elements in $S$ we set up a system of $|S|$ linear equations in $|S|$ unknowns where the unknowns are the logarithms. We can find this system of equations by applying part 2 of the algorithm to each element of $S$. The resulting system must be solved over the integers moctulo $2^{n}-1$. The number of iterations of part 2 to produce the recessary equations is approximately $|S| p(n, b)^{-1}$. For $G F\left(2^{127}\right)$ this quantity is minimized by $b=23$. Since there are 766,150 irreducible polynomials of degree at most 23 then we require this many equations. A random polynomial of degree at most 126 will factor into the database with $b=23$ with probability .000138 . This means that to produce the desired set of equations will require about $5,549 \times 10^{6}$ iterations of part 2 of the algorithm.

The nert section gives a variant of the Adleman algorithm which improves the situation for $G F\left(2^{127}\right)$.

\section{The Waterioo Algorithm}

This algorithm (see [3]) differs from the former algorithm in two ways. In part 2 where the polyaomial $h(x)=g(x) a^{a}(x)$ is factored this algorithm applies the extended Euclidean algorithm to the polynomials $h(x)$ and $f(x)\left(f(x)\right.$ is the irreducible polynomial of degree $n$ which defines $G F\left(2^{n}\right)$.) so that we can write

$$
h(x)=\frac{s(x)}{t(x)}
$$

where $(s(x), t(x))=1$ and deg $s(x)$, deg $t(x) \leq \frac{n}{2}$. One observation we can make at this point is that every polynomial $h(x)$ of degree at most $n$ ( $n$ add) can be written uniquely as a quotient of polynomials where each has degree at most $\frac{n}{2}$ and which are relatively prime. If both $s(x)$ and $t(x)$ factor into $s$ then $\log g(x)$ is readily computed by table lookup.

The advantage to this algorithm over the previous one is that it is more probable for two polynomials of degrees at most $\frac{n}{2}$ to factor into the database than it is for one polynomial of degree $n$. Let $N(b, i, j)$ be the number of pairs of relatively prime polynomials $(A(x), B(x))$ such that $\operatorname{deg} A(x)=i$, deg $B(x)=j$ and both are smooth with respect to $b$. (By smooth we mean that the polynomial factors into irrectucibles all of whose degrees are at most $b$.) For each irreducible polynomial $b(x)$ with degree $k \leq b$ define the enumerator of $b(x)$ by 


$$
\left(1+y^{k}+y^{2 k}+\cdots+z^{k}+z^{2 k}+\cdots\right) .
$$

Letting $l_{k}$ be the number of irrectucible polynomials of degree $k$ ve obtain the generating function for the $N(b, i, j)$ as

$$
\begin{aligned}
F(y, z) & =\prod_{k=1}^{b}\left(\sum_{i=0}^{\infty} y^{k}+\sum_{i=1}^{\infty} z^{k k}\right)^{I(k)} \\
& =\prod_{k}\left[\left(1-y^{k} z^{k}\right)\left(1-y^{k}\right)\left(1-z^{k}\right)\right]^{I(k)} \\
& =E(y) E(z) / E(y z)
\end{aligned}
$$

where $E(y)$ is the generating function for one smooth polynomial. The probability that an ordered pair of relatively prime polynomials $(A(x), B(x))$ each of degree at most $\frac{n}{2}$ are both smooth with respect to $b$ is

$$
p^{*}(n, b)=\left\{\sum_{a=1, j=\frac{\pi}{2}} N(b, i, j)\right\} 2^{n}
$$

In the case of $G F\left(2^{127}\right)$ Coppersmith [6] has eviluated this expression for $b=17$ and found $p^{*}(127,17) \equiv \frac{1}{7000}$. In order to simplify caiculations we approrimate $p^{*}(n, b)$ by $[p(n, b)]^{2}$. For $G F\left(2^{127}\right)$, $\left[p(127,17]^{2} \cong \frac{1}{5000}\right.$.

In the following table we list these probabilities for $b$ ranging between 1 and 30 and also we list the probabilities associated with the Adleman algorithm. The table also inciudes the expected number of iterations to produce enough equations to construct the database for each value of $b$ in the given range.

We see from the table that the number of iterations for the database is minimized by $b=20$. For our implementation we selected $b=17$ in order to keep the system of equations manageable.

The second difference in the Waterloo algorithm is in producing the equations for the database. We did not rely entirely on Part 2 of the algorithm. A number of equations were readily obtained by several techniques which make use of the fact that squaring is a linear operator in the field. Our principle tectnique here is referred to as the generation of systematic equations.

We briefly describe this technique with regard to $G F\left(2^{127}\right)$ generated by $f(x)=x^{127}+x+1$. Since $f(x)$ divides $x^{128}+x^{2}+x$, it is easily shown that $x^{y}, 0 \leq i \leq 126$, can be written as $e=\sum_{j=0}^{b} \gamma_{j} x^{y}$ where $\gamma_{j} \in\{0,1\}$ and so the $\log$ of any element of the form $e$ can be readily found. Similarly the log of any element of the form $\gamma-1+\sum_{j=0} \gamma_{f} x^{y}$ where $\gamma_{j} \in\{0,1\},-1 \leq j \leq 6$ can be found. This gives 31 equations where the maximum do gree is 16. Related to this idea is the observation that if $u(x)$ is any irrectucible polynomial of degree $d$ then the degrees of all irreducible polynomials of $w(u(x))$ are divisible by $d$. Using this method we obtained 142 linearly independent equations involving the 226 logarithms of irrecucible polynomials of degree $\leq 10$. 
Probability and expected number of runs for the new and Adlexan algorithm, $n=127$.

\begin{tabular}{|c|c|c|c|c|c|}
\hline Degree & $\begin{array}{l}\text { Total no. of } \\
\text { irred. polys }\end{array}$ & Probability & $\begin{array}{l}\text { Igorithm } \\
\text { Expected no. } \\
\text { of runs }\end{array}$ & Probability & $\begin{array}{l}\text { algorithm } \\
\text { Expected no. } \\
\text { of runs }\end{array}$ \\
\hline 1 & 1 & $1.27141469 \mathrm{E}-32$ & $7.8652544 \mathrm{E}+31$ & $0.47772090 \mathrm{E}-34$ & $0.20932720 E+35$ \\
\hline 2 & 2 & $1.61023467 E-30$ & $1.24205499 E+30$ & $0.10391370 E-32$ & $0.19246730 \mathrm{E}+34$ \\
\hline 3 & 4 & $1.42003032 E-27$ & $2.81684126 E+27$ & $0.10686600 \mathrm{E}-30$ & $0.37430020 E+32$ \\
\hline 4 & 7 & $1.15313844 \mathrm{E}-24$ & $6.07038995 E+24$ & $0.16022050 \mathrm{E}-28$ & $0.43689770 \mathrm{E}+30$ \\
\hline 5 & 13 & $3.46321796 E-21$ & $3.75373429 E+21$ & $0.12806100 E-25$ & $0.10151400 E+28$ \\
\hline 6 & 22 & $2.43083741 E-18$ & $9.05037906 E+18$ & $0.60489980 E-23$ & $0.36369650 E+25$ \\
\hline 7 & 40 & $1.75009226 \mathrm{E}-15$ & $2.28559379 E+16$ & $0.58661830 E-20$ & $0.68187380 \mathrm{E}+22$ \\
\hline 8 & 70 & $2.99279106 \mathrm{E}-13$ & $2.33895379 E+14$ & $0.20402690 E-17$ & $0.34309190 E+20$ \\
\hline 9 & 126 & $2.39477444 \mathrm{E}-11$ & $5.26145586 E+12$ & $0.40463370 \mathrm{E}-15$ & $0.31139250 \mathrm{E}+18$ \\
\hline 10 & 225 & $7.73424039 E-10$ & $2.90914154 E+11$ & $0.31781350 E-13$ & $0.70796220 \mathrm{E}+16$ \\
\hline 11 & 411 & $1.42517804 E-08$ & $2.88385022 E+10$ & $0.13612270 E-11$ & $0.30193290 E+15$ \\
\hline 12 & 746 & $1.48157461 E-07$ & $5.03518348 E+09$ & $0.29555160 E-10$ & $0.25240930 \mathrm{E}+14$ \\
\hline 13 & 1376 & $1.0660927 \mathrm{E}-06$ & $1.29069451 E+09$ & $0.41129290 \mathrm{E}-09$ & $0.33455440 E+13$ \\
\hline 14 & 2537 & $5.46899677 \mathrm{E}-06$ & 463887639 & $0.37461550 E-08$ & $0.67722710 \mathrm{E}+12$ \\
\hline 15 & 4719 & $2.19028276 \mathrm{E}-05$ & 215451634 & $0.24991280 E-07$ & $0.18882570 E+12$ \\
\hline 16 & 8799 & $7.12191038 \mathrm{E}-05$ & 123548311 & $0.12723630 \mathrm{E}-06$ & $0.69154690 \mathrm{E}+11$ \\
\hline 17 & 16509 & $1.96662481 E-04$ & 83945854.4 & $0.52428530 \mathrm{E}-06$ & $0.31488570 \mathrm{E}+11$ \\
\hline 18 & 31041 & $4.71015488 E-04$ & 65902291.5 & $0.17992970 \mathrm{E}-05$ & $0.17251720 E+11$ \\
\hline 19 & 58635 & $1.00764675 E-03$ & 58190035.4 & $0.53284450 E-05$ & $0.11004140 E+11$ \\
\hline 20 & 111012 & $1.96260912 E-03$ & 56563479.1 & $0.13895770 \mathrm{E}-04$ & $0.79888990 \mathrm{E}+10$ \\
\hline 21 & 210870 & $3.54177251 E-03$ & 59537985.4 & $0.32587350 \mathrm{E}-04$ & $0.64709140 \mathrm{E}+10$ \\
\hline 22 & 401427 & $5.96621694 E-03$ & 67283339.5 & $0.69740460 \mathrm{E}-04$ & $0.57560100 E+10$ \\
\hline 23 & 766149 & $9.45338803 E-03$ & 81044911.9 & $0.13806940 E-03$ & $0.55490060 \mathrm{E}+10$ \\
\hline 24 & 1465019 & .0142135998 & 103071637 & $2.25535770 \mathrm{E}-03$ & $0.57373450 E+10$ \\
\hline 25 & 2807195 & .0204564492 & 137227872 & $0.44523690 \mathrm{E}-03$ & $0.63048900 \mathrm{E}+10$ \\
\hline 26 & 5387990 & .0283794933 & 189855046 & $0.73751900 E-03$ & $0.73054240 E+10$ \\
\hline 27 & 10358998 & .0381757153 & 271350462 & $0.11680410 \mathrm{E}-02$ & $0.88686300 \mathrm{E}+10$ \\
\hline 28 & 19945393 & .0500248252 & 398709899 & $0.17773340 \mathrm{E}-02$ & $0.11222780 \mathrm{E}+11$ \\
\hline 29 & 38458183 & .0640949758 & 600018684 & $0.26095230 \mathrm{E}-02$ & $0.14737250 E+11$ \\
\hline 30 & 74248450 & .0805164802 & 922152208 & $0.33185230 E-01$ & - \\
\hline
\end{tabular}

Another example of making use of the linearity of squaring is a method referred to as weight manipulation [4] (for more details see [5]). Define $p(x)=x^{m}+g(x)$ where $g(x)$ has degree $k<<m$. Define $d$ to be the largest integer such that $2^{d} m \leq n$. Let $s=n-2^{d} m$ and consider

$$
\ell(x)=r_{p} p(x)^{2}(\bmod f(x)) \text {. }
$$

It follows that

$$
\operatorname{deg} \ell(x)=\max \left\{k 2^{d}, \operatorname{deg}\left(f(x)+x^{n}\right)\right\} .
$$

If $p(x)$ and $\ell(x)$ are smooth with respect to $b$ then we obtain an equation. For example, in $G F\left(2^{127}\right)$ generated by $f(x)=x^{127}+x+1$ take $p(x)=1+x^{7}$ and $d=7$ then $s=15$. This gives

$$
\ell(x)=x^{15}\left(1+x^{7}\right)^{16}=1+x+x^{15}
$$

Clearly, both $\ell(x)$ and $p(x)$ are smooth with respect to $b=17$. A similar result can be developed for 
$(p(x))^{2^{+1}}$

Coppersmith [6] has extended the idea behind these techniques and has obtained atriking improvements in the index calculus methods. We should mention that asymptotically the Waterloo aigorithm is of the same order $\left(e x p\left(c_{2}(n \log n)^{y}\right)\right)$ as the Adleman algorithm but for fields $G F\left(2^{n}\right)$ of practical interest it gives much better running times. The Coppersmith algorithm is described in the nert section.

The database for $G F\left(2^{127}\right)$ was constructed at Deneloor using their HEP computer [12]. A HEP consists of from one to sixteen process execution motules and each is a pipelined procestor with a depth of 8 . Since the HEP can run a gives program in parallel with itself, the inder-calculus algorithms are ideally suited to this architecture. Several copies of the algorithm were run in parallel to produce linear equations which when added to those found systematically and by weight manipulation would yield 16,510 linearly indepen. dent equations in the 16,510 unknown logs. Sirteen copies of the algorithm in parallel appeared to be optimal. The database took about 7 hours to build and computing individual logs using Part 2 takes under 1 second on the HEP. Having the database our implementation on a VAX11/780 takes about five minutes per logarithm.

As mentioned in the previous paragraph, the inder calculus algorithms are ideally suited to parallel processing. If we run $n$ copies of the program in parallel then the logarithm is obtained from the copy which finishes first. We compute the expected number of iterations to find the logarithm of an element given that $n$ copies of the algorithm are running simultaneously. Let $p$ be the probability that an iteration will succeed in computing the logarithm and let $q=1-p$. Suppose $i$ of the processes complete on the $k^{\text {th }}$ iteration and the remaining $n-i$ do not. The probability of this event is

$$
\left(p q^{k-1}\right)^{l}\left(1-\sum_{j=1}^{k} p q^{s-1}\right)^{n-i}
$$

and the probability that at least one of the processes finishes on the $k^{\text {th }}$ iteration is

$$
\sum_{i=1}^{n}\left(\sum_{i}^{n}\right)\left[p q^{k-1}\right]^{i}\left[1-\sum_{i=1}^{k} p q^{s-1}\right]^{n-1}
$$

Since $p \sum_{k=1}^{k} q^{s-1}=1-q^{k}$, (*) equals $q^{(k-1) n}\left[1-q^{n}\right]$. To compute the expected number of iterations we evaluate

$$
\sum_{t=1}^{\infty} k q^{(k-1) n}\left[1-q^{n}\right]=\left(1-q^{n}\right)^{-1}
$$

Since $q=1-p$ then it follows that $\left(1-q^{n}\right)^{-1} \cong(n p)^{-1}$ as one might expect. This expected aumber of iterations did occur when we made experimental runs on the HEP. 


\section{The Copperumith Atgortthm}

The algorithm described in this section makes ertensive use of the linearity of squaring in $G F\left(2^{n}\right)$. The method applies also to $p^{\text {th }}$ powers in $G F\left(p^{n}\right)$ for $n>1$.

Coppersmith's moctification [6] of this basic index-calculus algorithm of section 2 improves substantially the performance of both parts 1 and 2. Let's first consider part 1.

The algorithms of the previous sections and the algorithm given here rely cn the fact that polynomials of degree $k$ tend to factor into polynomials whose degrees are "umall". In order to generate exough equations to construct the database for $G F\left(2^{n}\right)$ select pairs of polynomials $(a(x), b(x))$ such that dega(x) $s d$ and $\operatorname{deg} b(x) \leq d$ where $d<b$ and the $g a d$ of $a(x)$ and $b(x)$ is 1 . It is easily seen that there are precisely $2^{2 d+1}$ such pairs. Let $k$ be a power of 2 near $\sqrt{n / b}$ and choose $h$ to be the least integer greater than $\frac{n}{k}$. Suppose also that the generating polynomial for the field has the form $f(x)=x^{n}+g(x)$ where $g(x)$ has low degree. Let $h(x)=x^{h k}=g(x) x^{h k-n}$ and define

$$
C(x)=x^{h} A(x)+B(x)
$$

and

$$
d(x)=[c(x)]^{k} .
$$

If both $c(x)$ and $d(x)$ are smooth with respect to $b$, then we obtain an equation for the database. In the case $n=127$ we take $b=17, k=4, h=32$ and $d=10$. The polynomials $c(x)$ and $d(x)$ have degrees $\leq 42$. Coppersmith [6] shows that the 2 million possible pairs $(a(x), b(x))$ yield about 47,000 equations in the 16,510 unknowns. The above procecture is a variant on the weight manipulation method of the previous section. A more direct application of weight manipulation can be described. We illustrate the technique in the case $G F\left(2^{127}\right)$ generated by $f(x)=x^{127}+x+1$. Select pairs of polynomials $(a(x), b(x))$ which are relatively prime and such that $\operatorname{deg} a(x) \leq 7$ and $\operatorname{deg} b(x) \leq 8$. Form the polynomials $c(x)=a(x) x^{31}+b(x)$ and $d(x)=x^{3}[c(x)]^{4}$ where deg $c(x) \leq 38$ and deg $d(x) \leq 35$. If $c(x)$ and $d(x)$ are smooth with respect to $b=17$ we get an equation.

We now describe part 2 of the algorithm. Let $g(x)$ be a field element whose log is to be found. Suppose $t=\operatorname{deg} g(x)$. Let $k$ be a power of 2 close to $\sqrt{n / t}$ and $h$ be the least integer greater than $n / k$. For ex. ample, if $n=127, b=17$ and $t=33$ then choose $k=2$ so that $h=64$. Finally select $d$ close to $(t+\sqrt{n / b}) \log n) / 2$. In our example we take $d=23$. Choose a selatively prime pair of polynomials $a(x)$ and $b(x)$ each of degree $\leq d$ such that $g(x)$ divides $c(x)=x^{h} a(x)+b(x)$. The choices for $a(x)$ and $b(x)$ can eavily be determined by solving a linear system of equations over $G F(2)$. Let $d(x)=[c(x)]^{k}$. If both $c(x)$ and $d(x)$ are smooth with respect to the bound $b^{(1)}=h \sqrt{b / n}$ then we have at most $2 r$ irrectucible factors of degree at most $b^{(1)}$. For each factor with degree $>b$ we iterate this procecture and rectuce the bound $b^{(1)}$ with each iteration. That is, at iteration $i, b^{(i)}=h(b / n)^{-2}$. If not both of $c(x), d(x)$ are amooth then hoose a new pair $a(x), b(x)$ and start again.

The asymptotic ruaging time of this algorithm is computed by Coppersmith [6] to be $\operatorname{cop}\left(e n^{\frac{1}{3}}(\log n)^{\frac{2}{3}}\right)$ which improves the results of the previous two sections. 
We have implemented part 2 of Coppersmith's algorithm. The following table displays test results of 8 samples each consisting of 100 randomly generated polynomials of degree at most 126 . The degree bound $b$ is abrays 17 but for eact sample we varied the Coppersmith degree bound $b^{\prime}$ and the value of $d$. We bave a column labelled "Total Number of Basic Iterations". This refers to the number of iterations required to obtain smoothness with respect to $b$ '. The column labelled "Total Number of Coppersmith Iteration" is the total number of iterations to obtain smoothness with respect to degree bound 17 for those polynomials with degree between 17 and $b^{\prime}$. We also found that in this case a single step recuction of the Coppersmith reduction was optimal.

\begin{tabular}{|c|c|c|c|c|}
\hline & & $\begin{array}{c}\text { Total } \\
\text { number } \\
\text { of basic } \\
\text { iterations }\end{array}$ & $\begin{array}{c}\text { Total } \\
\text { number } \\
\text { of } \\
\text { Coppersmith } \\
\text { iterations }\end{array}$ & $\begin{array}{c}\text { Average } \\
\text { time } \\
\text { in sec } \\
\text { per log }\end{array}$ \\
\hline 20 & 12 & 99,496 & 6,731 & 57 \\
\hline 20 & 13 & 72,519 & 7,262 & 44 \\
\hline 21 & 12 & 98,245 & 9,087 & 49 \\
\hline 21 & 13 & 39,327 & 9,995 & 19 \\
\hline 22 & 13 & 38,390 & 11,859 & 28 \\
\hline 22 & 14 & 24,741 & 16,299 & 28 \\
\hline 23 & 14 & 20,296 & 20,726 & 27 \\
\hline 23 & 15 & 12,857 & 27,045 & 47 \\
\hline
\end{tabular}

From the table it appears that $b^{\prime}=23$ and $d=14$ is optimal. With these values we ran a sample of 500 randomly generated polynomials with the following result. The column headings are the same as in the previous table.

\begin{tabular}{|l|l|l|l|l|}
\hline 23 & 14 & 120,661 & 101,563 & 26.6 \\
\hline
\end{tabular}

For $G F\left(2^{127}\right)$ we refer to an equation of the form

$$
[C(x)]^{4}=\left[A(x) x^{32}+B(x)\right]^{4}
$$

as a Coppersmith equation. As a direct generalization of the weight manipulation technique discussed earlier we consider equations of the forma

$$
x^{i}[C(x)]^{4}=x^{127-4^{4}}\left[A(x) x^{i}+B(x)\right]^{4}
$$

and refer to these as underfow equations. With the degree of $A(x)$ and $B(x)$ at most 9 in the Coppersmith equations we generated 19461 equations for the database in 8.1 hours on the VAX. With the degrees of $A(x)$ and $B(x)$ at most 8 we obtained 777 equations in 2.23 hours. The following table lists results when underflow equations were used. The columas labelled deg $A$ and deg $B$ refer to the bighest degree used for $A$ and $B$ respectively. For polyncmial $A$ we require that it has a constant term so that no Coppersmith equations are generated this way and the equations obtained from distinct rows are all different. 


\begin{tabular}{|c|c|c|c|c|}
\hline $\operatorname{deg} A$ & $\operatorname{deg} B$ & $i$ & $\begin{array}{c}\text { Value of } \\
\text { Equations }\end{array}$ & $\begin{array}{c}\text { Number of } \\
\text { (Hours) }\end{array}$ \\
\hline 8 & 9 & 31 & 7891 & 2.89 \\
\hline 9 & 8 & 30 & 7193 & 2.88 \\
\hline 10 & 7 & 29 & 5975 & 2.80 \\
\hline
\end{tabular}

If we use the Coppersmith equations with the degrees of $A(x)$ and $B(x)$ at most 8 and we use the underflow equations given in the first two rows of the table we get 22,861 equations in 8 hours or one equation every $.3499 \times 10^{-3}$ hours. This is approrimately a $16 \%$ improvement over collecting the 19461 equations using the Coppersmith equations with deg $A(x)$, and deg $B(x) \leq 9$. This data was obtained by running our program on a VAX11/780 at the University of Waterloo. The program is written in Fortran 77 with the ged routines in assembler.

\section{Conctusion}

We have described the basic index-calculus algorithm and two variants of it. The basic algorithm and the Waterloo algorithm both carry over to $G F(p), p$ a prime. Using the Euclidean algorithm for integers it is easy to show that given an integer $a, 1 \leq a \leq p-1$, that there erist integers $s$ and $t$ such that $a r=t(\bmod p)$ and $1 \leq|s|, t \leq p-1$.

As for $G F\left(2^{n}\right)$ it is clear that for $n=127$ this field is insecure and should be avoided in ayptographic scherses. In [13] Odlyzto analyses the performance of the Coppersmith aigorithm for various values of $n$ running on various types of equipment. The conclusion seems to be that an ambitious effort might be able to produce the necessary database for $n \leq 1280$.

\section{Referencen}

[1] L.M. Adleman, A suberponential algorithm for the discrete logarithm problem with applications to cryptography, Proc. 20th IEEE Found. Comp. Sci. Symp. (1979), 55-60.

[2] B. Arazi, Sequences constructed by operations modulo $2^{n}-1$ or mod $2^{n}$ and their application in evaluating the complexity of a $\log$ operation over $G F\left(2^{n}\right)$, preprint.

[3] I.F. Blake, R. Fuji-Hara, R.C. Mullin and S. A. Vanstone, Computing logarithms in finite fields of characteristic two, SLAM J. Alg. Disc. Methods, Vol. 5 2 (1984), 276-285.

[4] I.F. Blake, R. Fuj-Hara, R.C. Mullin and S.A. Vanstone, Finite field-techniques for shift registers with applications to ranging problems and cryptography, Final Report Project 106-16-02, Department of Communications (1983).

[5] I.F. Blake, R. Fuj-hara, R.C. Mullin and S. A. Vanstone, An attack on the discrete logarithm problem in $G F\left(2^{127}\right)$, Progress Report, Project $106-16-02$, Departecent of Communications (1982).

[6] D. Coppersmith, Fast evaluation of logarithms in fields of characteristic two, IEEE Trans. Inform. Theory. (July 1984), 587.594.

[7] W. Diffie and M.E. Hellman, New directions in cyptography, IEEE Trans. Inform. Theory. IT-22 (1976), 644-654. 
[8] T. EIGamel, A public key cryptosystem and a signature scheme based on discrete logarithms, IEEE Trans. Inform. Theory, to appear.

[9] T. Herlestam and R. Johameson, On computing logarithms over GF( $\left.2^{p}\right), B I T 21$ (1981), 326-334.

[10] D.E. Knuth The Arr of Computer Programming: Vol. 2. Seminumerical Algorithus. 2nd ed. AddisonWesley 1981.

[11] D.L. Long and A. Wigderson, How discreet is the discrete log? Proc. ISth ACM Symp. Theory of Compuring (1983), 413-420.

[12] R.C. Mullin, E. Neneth and N. Weidenhofer, Will public key cypto systems live up to their expectations? HEP implementation of the discrete $\log$ codebreaker, preprint.

[13] A. Odlyzko, Discrete logarithms in finite fields and their cyptographic significance, Eurocrypt-84 (to appear).

[14] S.C. Pohlig and M. Hellman, An improved algorithm for computing logarithms over $G F(p)$ and its cryptographic significance, IEEE Trans. Inform. Theory IT-24 (1978), 106-110.

[15] B.P. Schanning, Data encryption with public key distribution, EASCON Conf. Rec., Washington D.C., October 1979, 653-660.

[16] A.E. Western and J.C.P. Millex, Tables of indices and primitive roots, Royal Society Mathematical Tables. Cambridge University 9 (1968).

[17] K. Yiu and K. Peterson, A single-chip VLSI implementation of the diucrete exponential public key distribution system, Proc. GLOBECOM-82, IEEE (1982), 173-179. 